\title{
ADAPTIVE DECOUPLING CONTROL FOR BINARY DISTILLATION COLUMN INTO A 3D VIRTUAL ENVIRONMENT
}

\author{
Juan Sebastián Useche-Castelblanco* - Darío Amaya-Hurtado - Oscar Javier Reyes-Ortiz
}

Facultad de Ingeniería, Universidad Militar Nueva Granada, Bogotá, Colombia.

\begin{tabular}{|c|c|}
\hline ARTICLE INFO & Abstract: \\
\hline $\begin{array}{l}\text { Article history: } \\
\text { Received: } 21.6 .2019 . \\
\text { Received in revised form: } \\
\text { Accepted: } 1.2 .2020 .\end{array}$ & $\begin{array}{l}\text { The oil refinery plants have a particularly high energy } \\
\text { consumption in the distillation process. Several authors state that } \\
\text { these are the systems with the highest energy consumption in the } \\
\text { petrochemical industry. Currently, work is being done on the }\end{array}$ \\
\hline $\begin{array}{l}\text { Keywords: } \\
\text { Distillation column } \\
\text { MRAC Control } \\
\text { virtual environment. } \\
\end{array}$ & $\begin{array}{l}\text { implementation of adaptive controllers in order to improve the } \\
\text { efficiency of the distillation process and the quality of the product. } \\
\text { In this work the control of a binary distillation column developed } \\
\text { within a } 3 D \text { virtual laboratory is presented. Adaptive control by }\end{array}$ \\
\hline DOI: https://doi.org/10.30765/er.1480 & $\begin{array}{l}\text { reference model (MRCA) is used for the manipulation of bottom } \\
\text { and distillate concentrations. Initially, the mathematical } \\
\text { description of the process is obtained, together with the computer- } \\
\text { aided design, to subsequently implement the control technique. As } \\
\text { a result, the behavior of the controlled system in simulation is } \\
\text { shown and compared with the response of the embedded system } \\
\text { within the virtual environment. The main contribution of this work } \\
\text { is the interaction that a user can have with this type of processes } \\
\text { and the possibility of implementing modern control strategies } \\
\text { within this type of environments as a low-cost tool to support } \\
\text { education and training activities. }\end{array}$ \\
\hline
\end{tabular}

\section{Introduction}

The energy consumption used to obtain distilled products is considered to be one of the highest in petrochemical processes, it is known that the distillation units correspond to $3 \%$ of the energy consumption worldwide [1]. In the refining industries, new control techniques are being implemented to reduce the energy cost of the distillation towers and improve the quality of the product [2].

The control of distillation columns has been a research point in recent years. Methods have been developed to facilitate work with these multivariable systems such as decoupling techniques [3]. One of the modern controls applied to these systems are the adaptive controls that are usually used in processes where certain parameters are unknown and it is not possible to obtain with certainty the total model of the plant [4][5]. One of the most used types of adaptive control is the reference model (MRAC) that establishes a model to be followed by the plant according to its stabilization and behavior time. [6].

Some work related to adaptive controllers includes design of an adaptive predictive control strategy for an atmospheric distillation process of crude oil, where the methodology proposed to the coupled system reduces the complexity of the control structures [7]. Another is optimized adaptive control of an ideal reactive distillation column which demonstrated that the scheme is able to satisfactorily track the optimum operating point of the system [8]. Next is a non-linear adaptive controller for batch distillation; the controller with a simple structure and easy adjustment obtained good results in concentration control [9].

\footnotetext{
* Corresponding author

E-mail address: u1801739@unimilitar.edu.co
} 
The implementation of the controllers and their simulation are complemented by the development of digital platforms [10]. In this way, a person not only simulates a mathematical function, but it can also visualize its impact in a virtual model that it is similar to the real [11]. These virtual environments are used in order to give a better learning experience; decrease training costs and on-site testing [12].

Some examples of virtual laboratories as a complement to processes include high resolution virtual laboratory which determines performance for forming operations. This laboratory allows to work with the response in the microstructure of some models under different conditions [13]; Another examples are irtual laboratory for the simulation of the control of parallel robots, where the control response is studied with the dynamic singularities of the system [14]; and virtual laboratory for the design of thermoelectric material, where the behavior of different materials against different patterns is compared [15].

This paper shows the design and control of a binary distillation column within a virtual environment of the crude distillation process. It starts with the obtaining of the mathematical description and the estimation of the control parameters. To extend the use of this tool, the application is hosted inside a server and communication protocols are generated for the connection Matlab ${ }^{\circledR}$ - Unity $3 D \AA$. This for the user to not only see the result of the implementation of the controller, also can be able to implement their own systems of control.

\section{Methods and Materials}

The work is based on the development by parts and the final integration of the different elements that make up Figure 1. among which are the development of the virtual environment of the column, the implementation of the control technique and the protocol between Matlab ${ }^{\circledR}$ - Unity $3 \mathrm{D} \AA$.

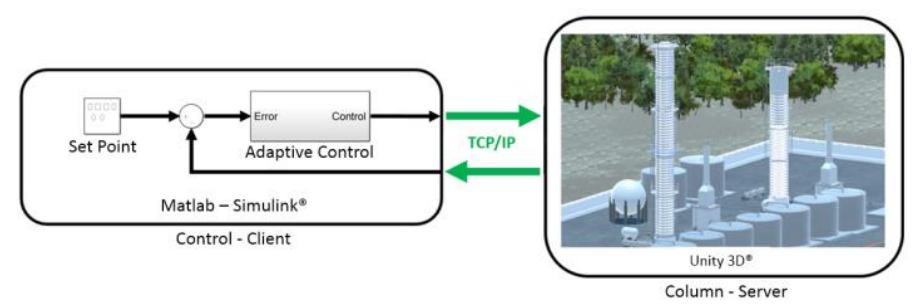

Figure 1. General scheme.

Next, the mathematical description of a binary distillation column of 43 plates is presented according to the scheme of Figure 2, the development of its CAD model, MRAC implementation and the link server between Matlab® - Unity $3 \mathrm{D} \AA$.

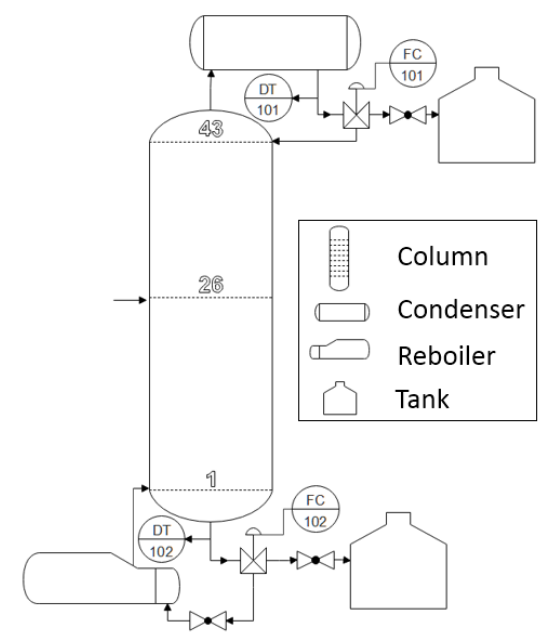

Figure 2. Crude distillation column. 


\subsection{Mathematical Description}

The distillation column is a system of $\mathrm{N}$ number of plates in which the crude is distilled at a defined temperature at atmospheric pressure. The binary distillation columns are systems with 3 inputs, the power supply and 2 for the control, the two outputs of the system are located at the top and bottom of the tower. [16]. The model starts by relating the contributions that are obtained by liquid, vapor and mass states of the crude in a plate $\mathrm{j}$. The matter diagram on a plate is shown in Figure 3.

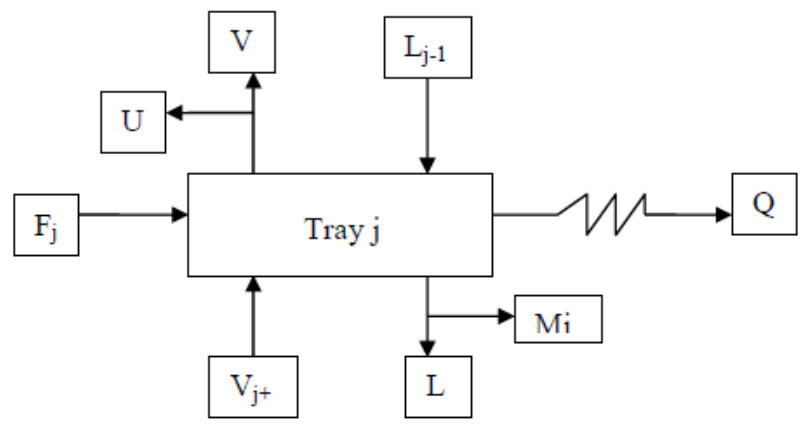

Figure 3. Analysis of the plate [16].

The development of the mathematical representation of the column is done using the expressions [17], [18] and the theory of [19]. The material balance for a plate $\mathrm{n}$ of the column is given by equation number 1 . This expresses the flow dynamics assuming heat conservation.

$$
\frac{\mathrm{dM}_{n}}{\mathrm{dt}}=L_{n+1}+\mathrm{V}_{n-1}-L_{n}-\mathrm{V}_{n}
$$

$\mathrm{M}_{n}=\mathrm{Ml}_{n}+\mathrm{Mv}_{n}$ is the addition of matter in liquid and vapor plate. $L_{n+1}$ is the liquid flow coming from the upper plate. $L_{n}$ the liquid flow that leaves the plate. $V_{n-1}$ the vapor flow coming from the lower plate. $V_{n}$ the flow of vapor that leaves the plate. As the states have a specific composition of the product, in equation 2 , the dynamics of the system are observed taking into account the molar fractions.

$$
\frac{\mathrm{dM}_{n} x_{n}}{\mathrm{dt}}=L_{n+1} x_{n+1}+\mathrm{V}_{n-1} y_{n-1}-L_{n} x_{n}-\mathrm{V}_{n} \mathrm{y}_{n}
$$

$\mathrm{M}_{n} x_{n}$ is equal to $\mathrm{Ml}_{n} x_{n}+\mathrm{Mv}_{n} \mathrm{y}_{n}, x_{n}$ is the mole fraction of the liquid on the plate, $\mathrm{y}_{n}$ the mole fraction in the plate of vapor. The balance is equal to $\frac{\mathrm{dM}_{n} x_{n}}{\mathrm{dt}}$ when making some considerations to the energy balance, equation 3 is shown.

$$
\frac{\mathrm{d} U_{n}}{\mathrm{dt}}=L_{n+1} h l_{n+1}+\mathrm{V}_{n-1} h v_{n-1}-L_{n} h l_{n}-\mathrm{V}_{n} h v_{n}
$$

Where $h$ expresses the different enthalpies of the plates and in the two states of the material. The energy is expressed as the composition of the energies of the two states as expressed in equation 4

$$
U=\mathrm{M}_{n} U_{n}=\mathrm{Ml}_{n} U l_{n}+\mathrm{Mv}_{n} U v_{n}
$$

Assuming that the vapor flow is the same in different plates, it is defined that molarity varies steadily. From the above, the conditions of equation 5 are defined.

$$
\mathrm{V}_{n}=\mathrm{V}_{n-1}, \frac{\mathrm{dMv}_{n} U v_{n}}{\mathrm{dt}}=0, \frac{\mathrm{dMv}_{n}}{\mathrm{dt}}=0
$$


Equation 6, 7 and 8 show the 3 expressions that define the binary system. The upper plate that has no liquid inlet, the intermediate plate that has an external product supply $F z_{f}$ and finally the bottom of the column that does not have a vapor inlet.

$$
\begin{gathered}
\frac{\mathrm{dM}_{n t} x_{n t}}{\mathrm{dt}}=\mathrm{V}_{n t-1} y_{n t-1}-L_{n t} x_{n t}-\mathrm{V}_{n t} \mathrm{y}_{n t} \\
\frac{\mathrm{dM}_{n f} x_{n f}}{\mathrm{dt}}=L_{n f+1} x_{n f+1}+\mathrm{V}_{n f-1} y_{n f-1}-L_{n f} x_{n f}-\mathrm{V}_{n f} \mathrm{y}_{n f}+F z_{f} \\
\frac{\mathrm{dM}_{1} x_{1}}{\mathrm{dt}}=L_{2} x_{2}-L_{1} x_{1}-\mathrm{V}_{1} \mathrm{y}_{1}
\end{gathered}
$$

Concentration $y_{n}$ which is expressed in equation 9, depends of the relative volatility constant of the mixture $(\alpha)$ and of the plate liquid concentration.

$$
y_{n}=\frac{\alpha x_{n}}{1+(\alpha-1) x_{n}}
$$

For the model linearization, the liquid and vapor differentials presented in the column are taken and these are equalized throughout the system as expressed in equation 10. These are taken as the equilibrium points of the system.

$$
d L_{n}=d L ; d V_{n}=d V
$$

The constant $K_{n}$ is presented in equation $11 . K_{n}$ is obtained by deriving equation 9 . This variable represents the relation between the differentials of liquid and vapor molar fractions $\left(d x_{n}\right.$ and $\left.d y_{n}\right)$.

$$
K_{n}=\frac{d y_{n}}{d x_{n}}=\frac{\alpha}{\left(1+(\alpha-1) x_{n}\right)^{2}}
$$

The mathematical expression that relates the amount of matter and the concentrations in the different plates using previously expressed equations is shown in equation 12 .

$$
d M_{n} d x_{n}=d L d x_{n+1}-\left(d L+K_{n} d V\right) d x_{n}+K_{n-1} d V d x_{n}+\left(x_{n+1}-x_{n}\right)-\left(y_{n}-y_{\mathrm{n}-1}\right) \mathrm{dV}
$$

A state space system is defined as shown in equation 13 .

$$
\left\{\begin{array}{c}
\dot{x}=A x+B u \\
y=C x
\end{array}\right\}
$$

Where $x=[x(i) \ldots x(n+1)]^{T}$ is the composition in the plates; $u=[V, L]^{T}$ are the input manipulated variables and $y=\left[x_{D}, x_{B}\right]^{T}$ are the controlled output variables (Distillate and Bottom concentrations).

To reduce the expression to a system of $2 \times 2$ with distillate and condensate concentrations, ithe presented has been implemented, by Samyudia Lee and Cameron in [20] defining equation 14.

$$
\left[\begin{array}{l}
d x_{D} \\
d x_{B}
\end{array}\right]=G_{L V}(s)\left[\begin{array}{l}
V \\
L
\end{array}\right]
$$


$G_{L V}(s)$ is calculated with the product of the state matrices $G_{L V}(s)=-C A^{-1} B$

These large-scale systems have a time delay. This occurs by the time it takes for the fluid or the vapor to move between the bottom and the crown of the column. To model this, the system is multiplied by a first-order function as illustrated in equation 15 [21].

$$
\left[\begin{array}{l}
d x_{D} \\
d x_{B}
\end{array}\right]=\frac{1}{1+\tau_{c} s} G_{L V}(s)\left[\begin{array}{l}
V \\
L
\end{array}\right]
$$

$\tau_{c}$ is calculated as illustrated in 16. $\tau_{c}$ involves the quantities of matter that are inside the column and outside it in the condenser or reboiler.

$$
\tau_{c}=\frac{M_{I}}{I_{S} \ln s}+\frac{M_{D}\left(1-d x_{D}\right) d x_{D}}{I_{S}}+\frac{M_{B}\left(1-d x_{B}\right) d x_{B}}{I_{S}}
$$

Where the variables represent the amount of liquid in the column $\left(M_{I}\right)$, condenser $\left(M_{D}\right)$ and in the reboiler $\left(M_{B}\right) ; I_{S}$ is the sum of impurities and $\mathrm{S}$ the factor of plates separation [22].

\subsection{CAD Model}

The model is done with SolidWorks ${ }^{\circledR}$ and using Blender ${ }^{\circledR}$ to import the column in the virtual environment. The column is developed with the regulations and dimensions exposed by Geoff Barker in [23]. Figure 4 shows the binary column of 43 plates. In the tower, the plates distribution [24] are applied.

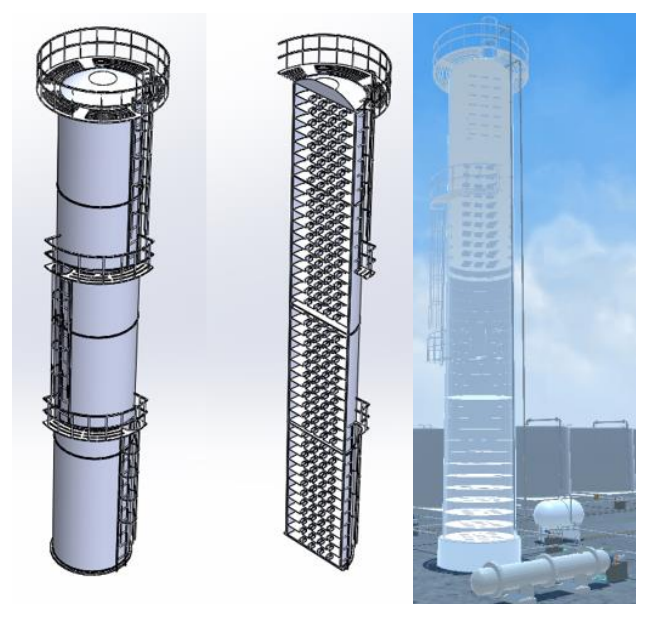

Figure 4. Binary Column.

The column is exported to the virtual environment and it is assembled with the complementary elements such as the condenser, the reboiler, the control valves and the pipelines.

\subsection{MRAC MIMO}

In the mathematical expression of equation 15 replacements are made based on [25] and [26]. Then the $\mathrm{Z}$ transformation is applied to the system functions with $\mathrm{T}=1$ seconds. Their difference in equations are extracted for its programming in the environment. The transformed functions and the equations are shown below. 


$$
\begin{gathered}
{\left[\begin{array}{l}
X d(\mathrm{z}) \\
X b(\mathrm{z})
\end{array}\right]=\left[\begin{array}{cc}
\frac{0.76 z-0.76}{z-0.94} & \frac{-0.9 z+0.9}{z-0.95} \\
\frac{0.6 z-0.6}{z-0.91} & \frac{-1.34 z+1.34}{z-0.93}
\end{array}\right]\left[\begin{array}{l}
L(\mathrm{z}) \\
V(\mathrm{z})
\end{array}\right]} \\
P_{11}(k)=0.76 u_{1}(k)-0.76 u_{1}(k-1)+0.94 P_{11}(k-1) \\
P_{12}(k)=-0.9 u_{2}(k)+0.9 u_{2}(k-1)+0.95 P_{12}(k-1) \\
P_{21}(k)=0.6 u_{1}(k)-0.6 u_{1}(k-1)+0.91 P_{21}(k-1) \\
P_{22}(k)=-1.3 u_{2}(k)+1.3 u_{2}(k-1)+0.93 P_{22}(k-1)
\end{gathered}
$$

Where $\mathrm{Xd}$ and $\mathrm{Xb}$ represent the molar fraction of the distillate and the bottom. $\mathrm{L}$ is the liquid flow of the condenser (reflux) and $\mathrm{V}$ the vapor flow of the reboiler.

In order to control the binary distillation column, decoupling functions are implemented $D_{12}(z)$ and $D_{21}(z)$ in order to be able to compensate the interactions between the system variables and obtain independent control loops [27]. Equations 19, 20 and 21 show the definition of the separation functions, the independent control loops and the uncoupled model of the plant.

$$
\begin{gathered}
D_{21}(z)=-\frac{P_{21}(z)}{P_{22}(z)} \quad D_{12}(z)=-\frac{P_{12}(z)}{P_{11}(z)} \\
T_{11}(z)=P_{11}(z)+P_{12}(z) D_{21}(z) \\
T_{22}(z)=P_{22}(z)+P_{21}(z) D_{12}(\mathrm{z}) \\
{\left[\begin{array}{l}
X d(\mathrm{z}) \\
X b(\mathrm{z})
\end{array}\right]=\left[\begin{array}{cc}
T_{11}(z) & 0 \\
0 & T_{22}(z)
\end{array}\right]\left[\begin{array}{l}
u 1(\mathrm{z}) \\
u 2(\mathrm{z})
\end{array}\right]}
\end{gathered}
$$

For each control loop an adaptive controller is implemented with the reference model. The MRCA is performed based on the development of Lyapunov stability exhibited in [28] and its implementation for a control system in [29].

The reference model and the process are expressed in the second order differential equations as shown in equations 22 and 23. The process is of a higher order but an adaptation to this behavior is sought and through the open-loop response of the system is identified that it is possible.

$$
\begin{gathered}
\ddot{y_{m}}+a_{1 m} \dot{y_{m}}+a_{0 m} y_{m}=b_{m} r \\
\ddot{y}+a_{1} \dot{y}+a_{0} y=b u
\end{gathered}
$$

The Lyapunov control law is the difference between the input signal and the output of the plant, multiplied by the parameter $\theta$. The error is the subtraction between the process and the reference model. The above is shown 24.

$$
u=\theta_{1} r+\theta_{2} y \quad e=y-y_{m}
$$

Deriving the error and replacing equations 22 and 23 as a function of the first derivatives, the following expression is obtained

$$
\dot{e}=\frac{\left(b u-\ddot{y}-a_{0} y\right)}{a_{1}}-\frac{\left(b_{m} r-y_{m}-a_{0 m} y_{m}\right)}{a_{1 m}}
$$


Replacing $y_{m}=e-y$ and $\ddot{y_{m}}=\ddot{e}-\ddot{y}$ in the previous equation, the system is expanded as shown in the equation below

$$
\frac{1}{a_{1 m}} \ddot{e}+\dot{e}+\frac{a_{m 0}}{a_{1 m}} e=\frac{b \theta_{1} r}{a_{1}}-\frac{b_{m} r}{a_{1 m}}-\frac{b \theta_{2} y}{a_{1}}-\frac{a_{0} y}{a_{1}}+\frac{a_{0 m} y}{a_{1 m}}-\frac{\ddot{y}}{a_{1}}+\frac{\ddot{y}}{a_{1 m}}
$$

When the plant model is adapted to the reference system constants are equal, in this way $a_{1}=a_{1 m}, a_{0}=$ $a_{0 m}, b=b_{m}$. Replacing these conditions in equation 26 and solving them, the differential equation 27 is obtained that expresses the behavior of the error in the adaptation.

$$
\dot{e}=-\frac{1}{a_{1 m}} \ddot{e}-\frac{a_{m 0}}{a_{1 m}} e+\frac{\left(b_{m} \theta_{1}-b_{m}\right)}{a_{1 m}} r-\frac{b_{m} \theta_{2}}{a_{1 m}} y
$$

A quadratic function is introduced, which derrival is shown in equation 28 to determine the Lyapunov stability and calculate the parameters of adaptation. The system is stable when the derivative of the function is negative.

$$
\frac{d V}{d t}=a_{1 m} e \frac{d e}{d t}+\frac{1}{\gamma}\left(b_{m} \theta_{1}-b_{m}\right) \frac{d \theta_{1}}{d t}+\frac{1}{\gamma}\left(b_{m} \theta_{2}\right) \frac{d \theta_{2}}{d t}
$$

With replacements in Equation 28, the error expression of 27, equation 29 is obtained.

$$
\frac{d V}{d t}=-a_{m} e^{2}+\frac{1}{\gamma}\left(b_{m} \theta_{1}-b_{m}\right)\left(\frac{d \theta_{1}}{d t}+\gamma r e\right)+\frac{1}{\gamma}\left(b_{m} \theta_{2}\right)\left(\frac{d \theta_{2}}{d t}-\gamma y e\right)
$$

The derivative is negative if the parameter values are updated so that the expressions are equal 0 . This is true and the derivative will be equal to $-a_{m} e^{2}$ when the following equations are met.

$$
\frac{d \theta_{1}}{d t}=-\gamma r e \quad \frac{d \theta_{2}}{d t}=\gamma y e
$$

Therefore, the value of the derivative is semi-defined, where error values and the parameters are limited. The value $\gamma$ can be varied to adjust the adaptation of the system. In this way the method solves the Lyapunov stability problems presented using gradients.

Applying the integral to the previous functions, the parameters for adaptation of the plant are as shown below

$$
\begin{gathered}
\theta_{1}=\frac{-\gamma_{1}}{b} \int r e d t+\theta_{1}(0) \\
\theta_{2}=\frac{\gamma_{2}}{b} \int y e d t+\theta_{2}(0)
\end{gathered}
$$

The reference model representing the desired behavior of the plant. Its design should take into account the response time in open loop and the following equations.

$$
M R=\frac{w_{n}{ }^{2}}{s^{2}+2 \rho w_{n} s+w_{n}{ }^{2}} \quad w_{n}=\frac{4.6}{\rho t_{s}}
$$


$\rho$ is the damping constant of the system, 4.6 represents the time constant, where the plant is within $1 \%$ error. The reference models of equations 34 and 35 are calculated with a $\rho=1$ and with open-loop response times of 200 s for the distillate and 300 s for the bottom respectively.

$$
\begin{aligned}
& M R_{d}=\frac{0.023^{2}}{s^{2}+2 * 1 * 0.023 s+0.023^{2}} \\
& M R_{f}=\frac{0.015^{2}}{s^{2}+2 * 1 * 0.015 s+0.0 .15^{2}}
\end{aligned}
$$

Figure 5 represents the complete system with the MRAC controller. The plant, decoupling functions, disturbances, reference models and controllers are observed.

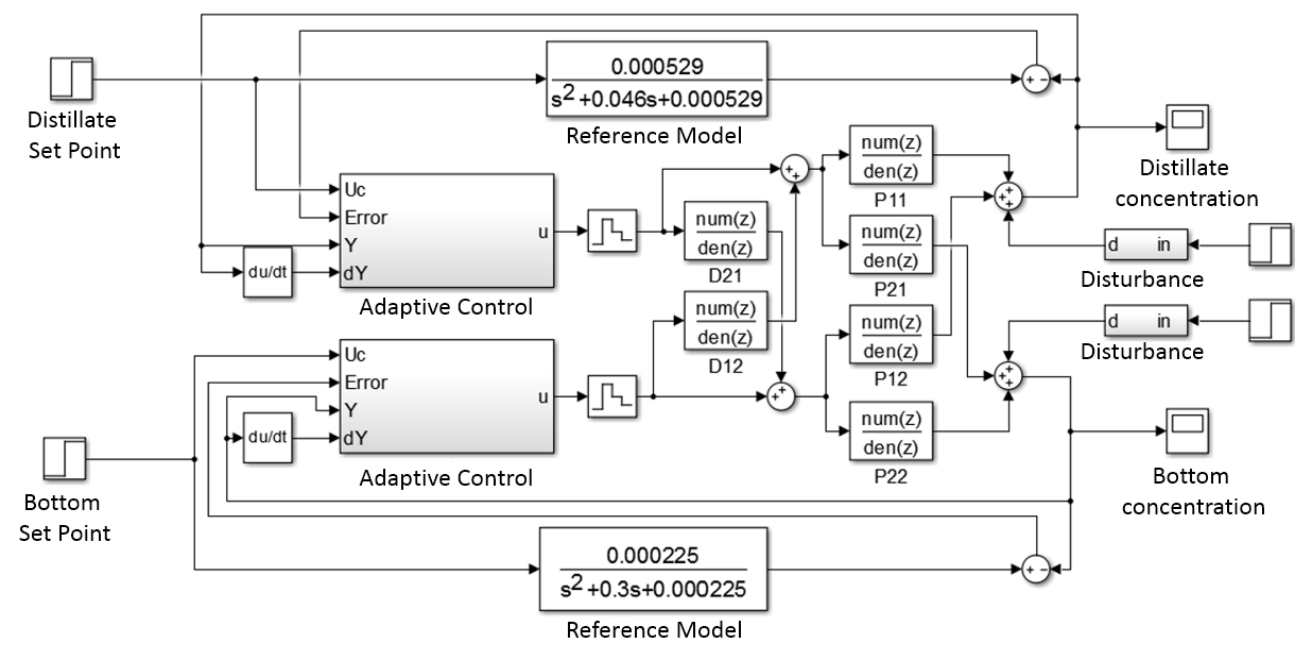

Figure 5. Scheme Plant-Controller.

\subsection{Server}

The virtual laboratory application is hosted on the server of the GAV research group of the Nueva Granada Military University. Through a Windows Server management protocol, the users are allowed to connect to the server, use the application remotely and connect it to their own Matlab ${ }^{\circledR}$. All the above is linked to a user profile with tracking and registration through a database already created by the research group. The user connection scheme is presented in Figure 6.

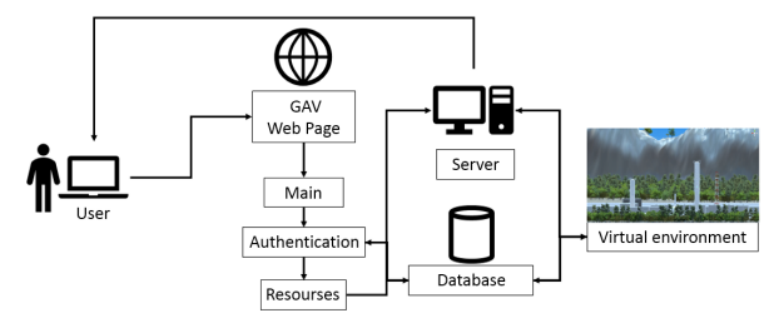

Figure 6. Server Connection Diagram.

The user's connection to the laboratory is divided into 3 stages. The first part is authentication, in the second one the user accesses to the server, and finishes with the opening of the port for the remote connection. 


\section{Results and discussion}

The control parameters are presented. The system response in simulation (Simulink $\left.{ }^{\circledR}\right)$ and the response in the virtual laboratory. On the other hand, the Pearson correlation applies between the graphs[30]. This parameter relates the covariance and the standard deviation of the data to determine the similarity between the responses in their dynamic state. The values used in the controller are shown in Table 1.

Table 1. Controller Parameters.

\begin{tabular}{|c|c|}
\hline Distillate controller & Values \\
\hline$\gamma_{1 / b}, \gamma_{2 / b}$ & $0.007,-0.01$ \\
\hline Bottom controller & Values \\
\hline$\gamma_{1 / b}, \gamma_{2 / b}$ & $-0.03,0.38$ \\
\hline
\end{tabular}

The above parameters were calculated to satisfy the stability of Lyapunov observed in equation 28 . These values comply with the equalities of equation 30 .

In Figure 7, the concentrations with their respective molar fraction references are observed within a range of 1,800 seconds.

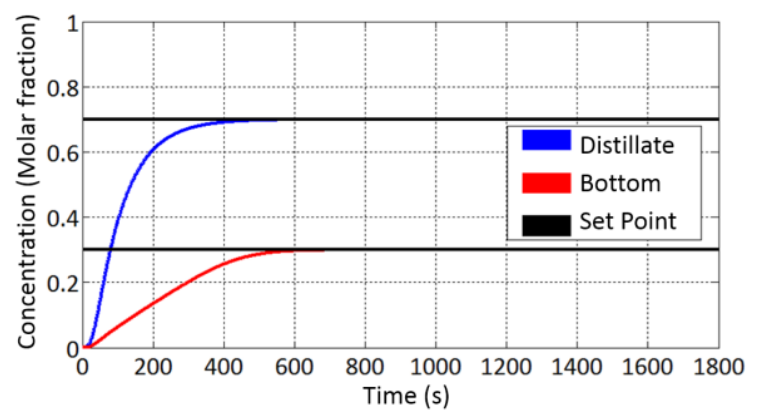

Figure 7. Controlled system response.

In Figure 8 the control signal for the different concentrations is observed. It can be seen being that a tracking system has a soft start system.

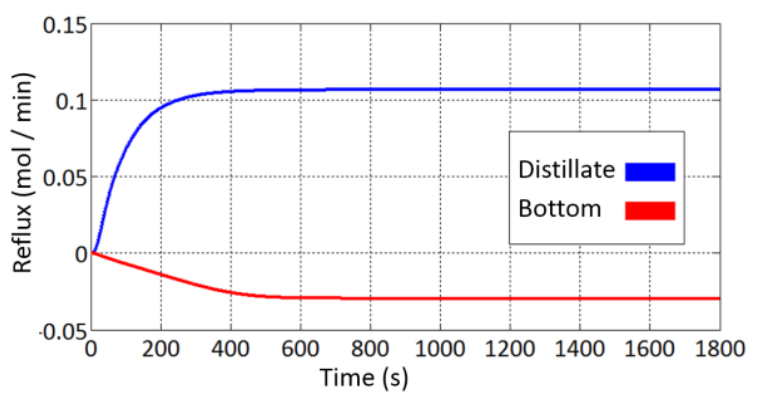

Figure 8. Control signal.

The above is due to how it is observed in Figure 9, the true reference of the plant is the model. This allows that the error between the plant and the model be low at the beginning. 


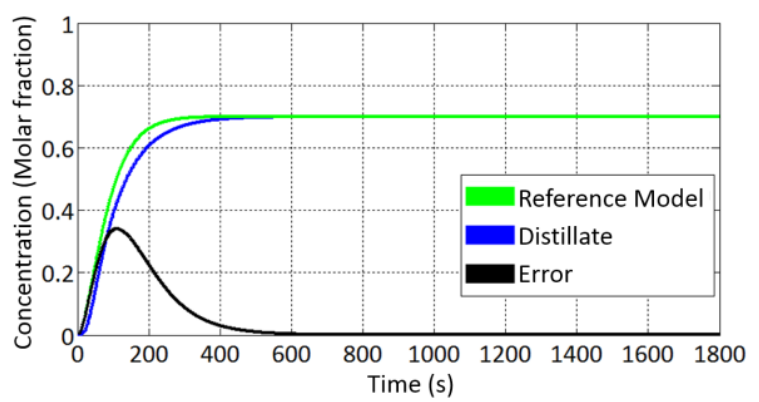

Figure 9. Model vs System.

Figure 10 shows the response of the different concentrations to a variable entry in the upper section of the column. It is observed by the functions of decoupling work not allowing the concentration of the bottom to be altered by the variation in the distillate

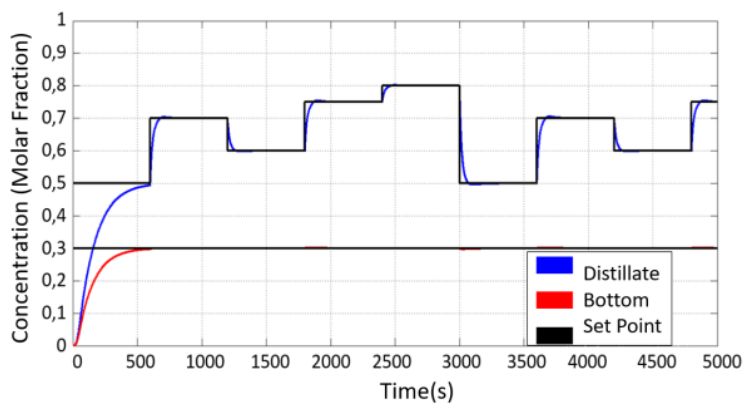

Figure 10. Controlled system response

Figure 11 shows the response of the column within the virtual environment. The light intensity of the control valve varies with the value of the control signal.

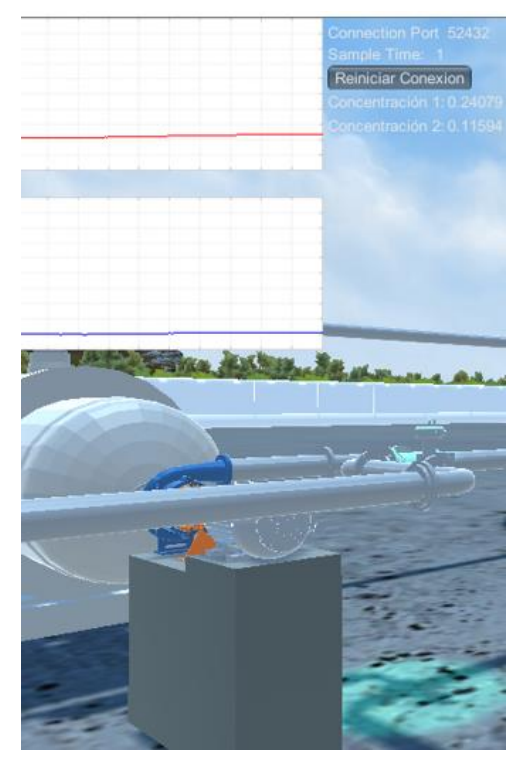

Figure 11. Control within the virtual environment.

Figure 12 shows the comparison of the concentration response in simulation and those obtained remotely in the virtual laboratory. Distillate concentration modifies the rise slope in the dynamic state. The bottom 
concentration is modified from critical to sub-damp. This is because the change in the dynamic response of the upper concentration must affect the decoupling equations calculated for the system.

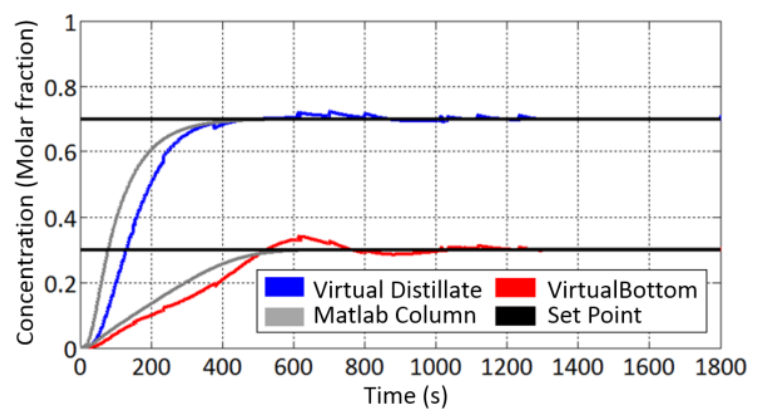

Figure 12. Comparative Responses of Binary Distillation Column.

Table 2 shows the consolidation of the comparison for the binary distillation column of the virtual laboratory and its local simulation in Matlab®.

Table 2. Consolidated.

\begin{tabular}{|c|c|c|c|}
\hline & Answer & Ts & Correlation between Graphics \\
\hline \multirow{2}{*}{ Distilled } & Virtual laboratory & $410 \mathrm{~s}$ & \multirow{2}{*}{$96.4 \%$} \\
\cline { 2 - 3 } & Simulink ${ }^{\circledR}$ & $400 \mathrm{~s}$ & \multirow{2}{*}{$97.2 \%$} \\
\cline { 2 - 3 } & Virtual laboratory & $900 \mathrm{~s}$ & \\
\cline { 2 - 3 } & Simulink ${ }^{\circledR}$ & $600 \mathrm{~s}$ & \\
\hline
\end{tabular}

On the other hand, from the previous graphs it is observed that the adaptive decoupling controller is susceptible to the transmission times, since it affects the decoupling equations of the previously calculated loops. The above is evidenced in a greater way by observing the stabilization time (Ts) for Bottom concentration. The correlation percentages obtained are above 95\% which shows that the response between the virtual plant and the simulated control in Simulink ${ }^{\circledR}$ is nearly the same.

\section{Conclusions}

The comparison of the MRAC with a diffuse PID worked on [31] which implements a similar mathematical description for the binary distillation column, demonstrating a faster and smoother response in the control signal. The adaptive controller with a variation of 0.08 in the bottom concentration has a stabilization time of 30 seconds less than the diffuse controller. Similarly, a reduction of 40 seconds in the concentration of distillate with a variation of 0.09 is obtained. In this way, it can be concluded that this type of adaptive controller works efficiently for this class of processes, helping to reduce energy expenditure without affecting the response time of the plant.

The implementation of the server that hosts the virtual laboratory proves to be necessary to reduce the computational resources of the user. The person only requires a computer with the ability to run Matlab®. The server is responsible for the graphic movement and the rendering of the different CAD models with the visualization properties granted. The results show that the implemented method only has a modification of 5\% in the correlation of the simulated response signals in Matlab®.

The reception and sending of data with the static laboratory has a loading and downloading speed of 8 $\mathrm{KB} / \mathrm{s}$ and $300 \mathrm{~KB} / \mathrm{s}$ respectively. When the user moves through space, the traffic goes to $300 \mathrm{~KB} / \mathrm{s}$ and 18 $\mathrm{MB} / \mathrm{s}$. To analyze this speed, the same rates were observed in common use pages where YouTube ${ }^{\circledR}$ live handles a traffic of $64 \mathrm{~KB} / \mathrm{s}$ of load and $7 \mathrm{MB} / \mathrm{s}$ of data download. Another example is seen in Skype ${ }^{\circledR}$ or TeamViewer ${ }^{\circledR}$ where download speeds range from 2 to $5 \mathrm{MB} / \mathrm{s}$. This shows that the rate of exchange and network resources required by the laboratory are medium if it considers that the laboratory handles 3D graphics, animations, access to server hardware and online control. 


\section{Acknowledgments}

Este producto es derivado de los proyectos, de alto impacto IMP-ING-2132 y de investigación INV-ING1911; financiados por la vicerrectoría de investigaciones de la Universidad Militar Nueva Granada, a la cual los autores agradecen.

\section{References}

[1] Shi B, Yang X, Yan L: Optimization of a crude distillation unit using a combination of wavelet neural network and line-up competition algorithm, Chinese J Chem Eng, 25 (2017), 8, 1013-1021.

[2] Yamashita AS, Zanin AC, Odloak D: Tuning the Model Predictive Control of a Crude Distillation Unit,ISA Trans, 60 (2015), 1-13.

[3] Dorrah HT, El-garhy AM: PSO based optimized fuzzy controllers for decoupled highly interacted distillation process, Ain Shams Eng, 3 (2012), 251-266.

[4] Selivanov A, Fradkov A, Liberzon D: Adaptive control of passifiable linear systems with quantized measurements and bounded disturbances «t,Syst Control Lett, 88 (2016), 62-67.

[5] Murlidhar GM, Jana AK: Nonlinear adaptive control algorithm for a multicomponent batch distillation column, Chem Eng Sci, 62 (2007), 1111-1124.

[6] Zhang S, Feng Y, Zhang D: Application Research of MRAC in Fault-Tolerant Flight Controller,Procedia Eng, 99 (2015), 1089-1098.

[7] Raimondi A, Favela-Contreras A, Beltrán-Carbajal F, et al: Design of an adaptive predictive control strategy for crude oil atmospheric distillation process, Control Eng Pract, 34 (2015), 39-48.

[8] Valluru J, Purohit J: Adaptive Optimizing Control of an Ideal Reactive Column,IFAC-PapersOnLine, (2015), 489-494.

[9] Jana AK: Synthesis of nonlinear adaptive controller for a batch distillation,ISA Trans, 46 (2007), 4957.

[10] Potkonjak V, Gardner M, Callaghan V, et al: Virtual laboratories for education in science, technology, and engineering: A review, Comput Educ, 95 (2016), 309-327.

[11] Dorado G, Dorado MP: Virtual laboratory on biomass for energy generation,J Clean Prod, 112 (2016), 3842-3851.

[12] Gorai P, Gao D, Ortiz B, et al: TE Design Lab : A virtual laboratory for thermoelectric material design,Comput Mater Sci, 112 (2016), 368-376.

[13] Zhang H, Diehl M, Roters F, Raabe D: A virtual laboratory using high resolution crystal plasticity simulations to determine the initial yield surface for sheet metal forming operations, Int J Plast, 80 (2016), 111-138.

[14] Peidró A, Reinoso O, Gil A, et al: A Virtual Laboratory to Simulate the Control of Parallel Robots,IFAC-PapersOnLine, 48 (2015), 019-024.

[15] Carpeño A, Contreras D, Lopez S: 3D virtual world remote laboratory to assist in designing advanced user defined DAQ,Fusion Eng Desing, (2016), 2-5.

[16] Gunorubon J, Diepriye O, State R: Simulation of a Multi-component Crude Distillation Column,Am J Sci Ind Res, 4 (2013), 4, 366-377.

[17] Useche-Castelblanco JS, Amaya D, Orjuela A: MPC MIMO State Space Control for Crude Oil Refining Process,Int J Control Theory Appl, 10 (2017), 21, 143-153.

[18] Mehta I, Singh V, Gadre V: Simulation and control of binary distillation column using XMOS, Int Conf Signal, Image Process Appl, (2011), 227-236.

[19] Marquardt W, Amrhein M: Development of a Linear Distillation Model from Design Data for Process Control,Comput Chem Eng, 18 (1994), Suppl, S349-S353.

[20] Samyudia Y, Lee PL: A methodology for multi-unit control desing,Chem Eng Sci, 49 (1994), 23, 3871-3882.

[21] Engineering C, This D, Hughes RR: The Dominant Time Constant for Distillation Columns, Comput Chem Eng, 11 (1987), 6, 607-617.

[22] Sigurd. S: Dynamics and control of distillation columns - a critical survey,Model Identif Control, 25 (1997), 5, 177-217.

[23] Barker G: The Engineer's Guide to Plant Layout and Piping Design for the Oil, Gulf Professional 
Publishing, 2018.

[24] Oladimeji T, Sonibare J: Environmental Impact Analysis of the Emission from Petroleum Refineries in Nigeria,Energy Environ Res, 5 (2015), 1, 33-39.

[25] Sivananaithaperumal S, Baskar S: Design of multivariable fractional order PID controller using covariance matrix adaptation evolution strategy, Arch Control Sci, 24 (2014), 2, 235-251.

[26] Useche-Castelblanco JS: Laboratorio Virtual para el Proceso de Destilación de Crudo,. Universidad Militar Nueva Granada, 2018.

[27] Valencia Palomo G: Aplicacion del Control Predictivo Multivariable a una Columna de Destilacion Binaria,. Centro Nacional de Investigacion y Desarrollo Tecnologico, 2006.

[28] Ganapathy R, Kumar V: Uniform ultimate bounded robust model reference adaptive PID control scheme for visual servoing,J Franklin Inst, 354 (2017), 1741-1758.

[29] Han J, Yu S, Yi S: Adaptive control for robust air flow management in an automotive fuel cell system,Appl Energy, 190 (2017), 73-83.

[30] Mu Y, Liu X, Wang L: A Pearson's correlation coefficient based decision tree and its parallel implementation,Inf Sci (Ny), 435 (2018), 40-58.

[31] Mishra P, Kumar V, Rana KPS: A fractional order fuzzy PID controller for binary distillation column control,Expert Syst Appl, 42 (2015), 8533-8549. 